\title{
Has Resveratrol a Modulatory Effect on Renal Injury Associating Diabetic Nephropathy in Albino Rats?
}

\author{
NERMEEN B. SADEK, M.D.*; MOHAMED A. ESHRA, M.D.*; EFFAT A. KHOWAILED, M.D.*; \\ LAILA A. RASHED, M.D.** and BASMA E. ABOULHODA, M.D.*** \\ The Departments of Physiology*, Biochemistry** and Anatomy, Faculty of Medicine, Cairo University, Egypt
}

\begin{abstract}
Background: The involvement of abnormally stimulated renin angiotensin system, excessive formation of reactive oxygen species, increased glucose metabolite flux and upregulation of pro-inflammatory and profibrotic cytokines in the pathogenesis of diabetic nephropathy has become an emerging concern. Exploration of novel therapies that specifically target such factors is a major goal in diabetes treatment.

Aim of Study: Our aim is to explore the potential protective and therapeutic effect of resveratrol on $\mathrm{DN}$ progression through its antioxidant, anti-inflammatory and antifibrotic role in comparison with losartan as one of the therapeutic regimens used in diabetic nephropathy.
\end{abstract}

Material and Methods: Forty eight male albino rats were assigned into six groups; control, diabetic nephropathy, prophylactic losartan, prophylactic resveratrol, therapeutic losartan and therapeutic resveratrol. Systolic blood pressure, fasting glucose, glomerular filtration rate, urea, urea albumin excretion, tumor necrosis factor a, tansforming growth factor-(3, interleukin- $\sigma$ and superoxide dismutase have been measured. Histopathological assessment of inflammation, glomerulosclerosis and fibrosis using hematoxylin and eosin, Masson's trichrome and Periodic acid shift staining and histomorphometric analysis for glomerular and tubular parameters have also been performed.

Results: Prophylactic resveratrol showed significant regression in inflammation, fibrosis and oxidative stress compared with its therapeutic effect.

Conclusion: Resveratrol showed better outcome compared with losartan with significant change in the biochemical parameters.

Key Words: Diabetic nephropathy - Resveratrol - Losartan, inflammation - Oxidative stress - Fibrosis.

\section{Introduction}

DIABETIC nephropathy (DN) is known as the leading cause of end-stage renal disease (ESRD)

Correspondence to: Dr. Nermeen B. Sadek, E-Mail: Nermeen.B.Sadek@kasralainy.edu.eg worldwide. Statistics show that $30 \%$ of patients with diabetes suffer from renal complications. The rapidly increasing prevalence of diabetes and its complications has become a major health problem especially with the worldwide epidemic of obesity and metabolic syndrome.

The development and progression of DN correlates closely with level of hyperglycemia and blood pressure. Previous studies postulated that effective glycemic control in diabetic patients decreased the risk of microvascular complications including DN. Most conventional drugs used for treatment of diabetes and its complications as DN depend on glycemic control and blood pressure lowering effect. However, such therapies do not prevent the progression of $\mathrm{DN}$ [2].

The pathogenesis of DN is an extremely complex and multifactorial process. Several pathways have been recognized to contribute to the development of DN including an abnormally stimulated renin angiotensin system (RAS), excessive formation of reactive oxygen species (ROS), increased glucose metabolite flux and increased proinflammatory and profibrotic cytokines $[3,4$, .

Inhibition of the renin angiotensin system (RAS) is the major concern for treatment DN. However, therapies that target the RAS, such as ACE inhibitors or angiotensin receptor-1 blockers, have been only partially successful as these drugs just delay the progression to ESRD [5].Therefore, there is an urgent need to recognize better targets to prevent and delay ESRD.

Resveratrol (RSV) is a biologically active polyphenol, produced in more than 70 plants species and found in high concentrations in grapes, red wine and peanuts [б]. 
Some studies have revealed that resveratrol administration improves glycemic control, insulin sensitivity and other metabolic parameters in diabetic rats and patients with T2DM [7,8]. Moreover, RSV attracted interest due to its anti-inflammatory, antioxidant, vasodilatation, and anti-aging properties [9]. These results suggested the use of resveratrol in addition to the usual therapies for diabetes.

On the other side, previous randomized clinical trial revealed that resveratrol supplementation was not useful in improving glycemic control, body weight or energy intake [10]. These contradictory results raise the need for further investigations of the effect of resveratrol as an adjuvant therapy for diabetes and its complications.

Therefore, our aim in the present work is to determine the potential protective and therapeutic effect of resveratrol on hyperglycemia, hypertension and diabetic nephropathy through its antioxidant, anti-inflammatory and antifibrotic role and to compare it with losartan as one of therapeutic regimens used in diabetic nephropathy.

\section{Patients and Methods}

\section{Experimental animals:}

The study was carried out on 48 adult male albino rats weighing $150-200 \mathrm{~g}$. Rats were inbred in the Experimental Animal Unit, Faculty of Medicine, Cairo University in 2016. The study was approved by the institutional Ethical Committee. All experimental procedures were carried out according to the standard guidelines for care and use of laboratory animals published by the Guide to the Care and Use of Experimental Animals (Vol. 1, 2nd ed., 1993, and Vol. 2, 1984, Canadian Council on Animal Care (CCAC). Animals had free access to food and water. They were kept at room temperature and normal dark-light cycles throughout the study.

\section{Materials:}

1- Streptozotocin powder (STZ) dissolved in fresh $0.05 \mathrm{M}$ citrate buffer, $\mathrm{pH}=4.5$ and immediately injected intraperitoneally (ip) as recommended by the National Institute of Diabetes Animal Models of Diabetic Complications Consortium.

2- Losartan $10 \mathrm{mg} / \mathrm{kg} /$ day dissolved in $1 \mathrm{ml}$ saline by oral gavage [11]

3- Resveratrol $10 \mathrm{mg} / \mathrm{kg} /$ day dissolved in $1 \mathrm{ml}$ saline by oral gavage [12]
Chemicals were purchased from Sigma (St. Louis, MO, USA).

\section{Animal groups:}

Rats were divided equally into 6 groups, 8 rats each as follows: (i) Control group, (ii) Diabetic nephropathy (DN) group received single ip injection of $50 \mathrm{mg} / \mathrm{kg} \mathrm{STZ}$ for induction of diabetes [13] Diabetes was confirmed by measuring fasting serum glucose 3 days after STZ injection. Rats with fasting glucose level above $250 \mathrm{mg} / \mathrm{dl}$ were considered diabetics.

After induction of diabetes, rats were received an equivalent volume of vehicle orally for 8 weeks. Nephropathy was confirmed by significant increase in protinuria and serum creatinine. (iii) Prophylactic losartan (LOS+DN) group received losartan, started at onset of diabetes and continued for 4 weeks, till the occurrence of nephropathy (the estimated duration for developing nephropathy is 4 weeks after induction of diabetes [14] and then they were left without any medications till the end of study. (iv) Prophylactic resveratrol (RSV+DN) group received resveratrol, started at onset of diabetes and continued for 4 weeks, till the occurrence of nephropathy and then they were left without any medications till the end of study. (v) Treated losartan (DN+LOS) group, received losartan for 4 weeks, started after the occurrence of diabetic nephropathy till the end of the study. (vi) Treated resveratrol (DN+RSV) group, received resveratrol for 4 weeks, started after the occurrence of diabetic nephropathy till the end of the study.

\section{Experimental protocol:}

At the end of the study, all rats were subjected to overnight fasting, blood samples were collected from the retro-orbital plexus for assessment of fasting serum glucose level, blood urea nitrogen (BUN), plasma creatinine for assessing glomerular filtration rate (GFR), tumor necrosis factor- a (TNFa), tissue growth factor- $\beta$ (TGF- $(3)$, interleukin- 6 (IL6) and superoxide dismutase (SOD). Urine samples were collected for measuring creatinine in urine and 24 hour urine were collected, for measuring GFR and urinary albumin excretion (UAE).

\section{Measurement of systolic blood pressure:}

Using the Power lab data acquisition system (AD instruments; Pty Ltd Australia); an electronic version of the traditional sphygmomanometer cuff method. 


\section{Biochemical assessment:}

Measurement of fasting serum glucose:

The serum glucose was assayed by the method adopted by Trinder.

\section{Determination of kidney functions:}

\section{1- Measurement of UAE using Albuwell M Kit:}

Murine Microalbuminuria ELISA.

Collection of 24 hours urine samples was done using specialized wire mesh metabolic cages. Before sampling, rats were deprived from food for 1 day with free access to water.

2- Measurement of serum creatinine and BUN using kits (Bioclin, Santa Coloma, Spain).

3- Determination of GFR was done by calculating the creatinine clearance:

Creatinine clearance $=$ (urine level of creatinine $x$ 24hours urine volume) + (plasma level of creatinine x 24 x 60).

\section{Determination of TNF- $a, T G F-(3$ and IL6:}

The concentrations of cytokines were measured by the sandwich Enzyme Linked Immuno-Sorbent Assay (ELISA) kits according to the manufacturer's instruction, (R\&D syst; Minneapolis; USA).

\section{Measurement of SOD:}

Superoxide dismutase (SOD) activity in kidney homogenate was measured through the inhibition of nitroblue tetrazolium (NBT) reduction by $\mathrm{O}_{2-}$ generated by the xanthine/xanthine oxidase system. One SOD activity unit was defined as the enzyme amount causing 50\% inhibition in $1 \mathrm{~mL}$ reaction solution per milligram tissue protein and the result was expressed as U/mg protein as previously described [15]

\section{Histopathological examination:}

Kidneys from all groups were dissected and fixed immediately in $10 \%$ formol saline. The specimens were then washed, dehydrated in ascending grades of ethanol, cleared in xylene and impregnated in paraffin. Sections of $5 \mu \mathrm{m}$ thickness were cut and subjected to hematoxylin and eosin (Hx\&E), Masson's trichrome (MT) and Periodic acid shift (PAS) staining.

\section{Histomorphometric analysis:}

All histomorphometric parameters were estimated via the Leica Imaging System (Leica Imaging System Ltd., Cambridge, England) image analyzer software program "Lecia Qwin 500C". The Leica Imaging System was first automatically calibrated to convert the measurement units (pixels) produced by the image analyzer program into actual micrometer units. Using the measuring field menu; the parameters to be measured were chosen and then, in each field, the tissue was enclosed inside the standard measuring frame measuring and the parameter of interest was masked by a blue binary color and measured. All measurements were estimated under magnification 400 inside the standard measuring frame $\left(85,550 \mu \mathrm{m}^{2}\right)$ in ten non-overlapping randomly-selected fields from ten sections of each animal by two independent observers blinded to the groups.

\section{For H\&E-stained sections:}

Interstitial cell infiltration score and vascular congestion area percent were determined. Interstitial cell infiltration scores were assessed by using semiquantitative measurements according to the proportion relative to the total section area and classified as follows: 0 (nil), 1 (<25\%), $2(25-50 \%)$, $3(50-75 \%)$, and 4 (>75\% of tubulointerstitial fields).

\section{For Masson's Trichrome-stained sections:}

The area percent of collagen fibers was measured using the measuring field menu; the area and area percentage were masked by blue binary color and estimated using the image analyzer.

\section{For PAS-stained sections:}

PAS-stained sections were used for semi-quantitative scoring of glomerulosclerosis as previously described [16] (grade 0, normal glomeruli; grade 1 , sclerotic area up to $25 \%$ (minimal sclerosis); grade 2 , sclerotic area 25 to $50 \%$ (moderate sclerosis); grade 3, sclerotic area 50 to $75 \%$ (moderatesevere sclerosis); grade 4, sclerotic area 75 to $100 \%$ (severe sclerosis). The glomerulosclerotic index (GSI) was then calculated according to the number of glomeruli in each grade of glomerulosclerosis [17].

\section{Statistical methods:}

Data were analyzed using statistical package SPSS version 24. Data were summarized using mean and standard deviation. Comparisons between groups were done using ANOVA with multiple comparisons post hoc test. $p$-values $<0.05$ were considered statistically significant.

\section{Results}

\section{Metabolic and hemodynamic state:}

Both, the fasting serum glucose level as a metabolic parameter and the systolic blood pressure (SBP) as a hemodynamic parameter were signifi- 
cantly increased in diabetic group compared to control group (Table 1).

Both, prophylactic resveratrol and losartan groups were associated with significant reduction in fasting serum glucose level and SBP level compared to DN group but with better outcome in resveratrol group, approaching their corresponding values in control group. There wasn't any significant difference between prophylactic resveratrol and losartan.

On the other hand, treating DN rats with resveratrol or losartan caused marked improvement in fasting serum glucose level and SBP level, but therapeutic resveratrol was better significantly than corresponding losartan in reducing hyperglycemia.

There was no significant difference between prophylactic and therapeutic resveratrol regarding serum glucose level and systolic blood pressure.

\section{Kidney function parameters:}

A significant deterioration $(p<0.05)$ was observed in the kidney function in DN group compared to control group manifested by increased urea and UAE and decreased GFR (Fig. 1). This deterioration was markedly improved $(p<0.05)$ in both prophylactic resveratrol and losartan groups, with a more non-significant $(p>0.05)$ improving effect in resveratrol prophylactic group compared to losartan prophylactic group.

The same beneficial effects occurred with both therapeutic groups as all kidney function parameters were significantly improved $(p<0.05)$ in relation to their corresponding values in untreated groups. Despite this beneficial effect, a significant difference still existed in respect to control group.

As it was obvious from (Fig. 1), there was no significant difference $(p>0.05)$ between the effect of resveratrol on urea, UAE and GFR to the corresponding effect of losartan in both prophylactic and treated groups, but still resveratrol had a better effect. Moreover, prophylactic resveratrol showed no significant difference in kidney functions in comparison with therapeutic resveratrol.

\section{Proinflammatory markers:}

DN rats showed significantly increased TNF$\alpha$ and IL6 compared to the control group (Fig. 2ab) $(p<0.05)$ indicating inflammatory changes in diabetic kidney, while prophylactic groups with both resveratrol and losartan were protected against such changes. These groups showed significantly decreased TNF $\alpha$ and IL6 compared to DN group $(p<0.05)$, remarkably prophylactic resveratrol showed significant difference $(p<0.05)$ compared to prophylactic losartan group regarding IL6.

It was also observed from fig. 1that both TNF $\alpha$ and IL6 were significantly decreased in treated groups compared to DN group ( $p<0.05)$, but these values were still significantly increased in comparison with the control group $(p<0.05)$. It is worth noting that prophylactic resveratrol significantly decreased $(p<0.05)$ IL6 compared to therapeutic resveratrol.

\section{Profibrotic and anti-oxidative markers:}

As observed in (Fig. 2c-d), DN group showed significant elevation in TGF- $\beta$ and significant reduction in SOD activity compared to control group $(p<0.05)$, thus indicating increased fibrosis and oxidative stress respectively, both contributing to the development of diabetic nephropathy. These parameters showed marked improvement after prophylactic resveratrol and losartan with more significant improvement in resveratrol prophylactic group as regards SOD activity $(p<0.05)$. At the same time, both treated groups showed significant reduction in TGF- $\beta$ level in contrast to significant increase in the antioxidant SOD activity relative to DN group $(p<0.05)$, with no significant difference between both groups and better outcome in resveratrol group. In prophylactic and treated groups, both parameters still showed significant difference in comparison with control group $(p<0.05)$. Remarkably, both TGF- $\beta$ and SOD significantly improved with prophylactic resveratrol compared to therapeutic resveratrol $(p<0.05)$.

\section{Histopathological results:}

\section{Examination of H\&E stained sections (Fig. 3):}

Examination of the DN group revealed statistically significant increase in interstitial cell infiltration and vascular congestion. Comparing losartan and resveratrol prophylactic and treatment groups demonstrated that pre-DN resveratrol resulted in the best improvement in those parameters.

\section{Examination of PAS stained sections (Fig. 4):}

Diabetic rats displayed features of glomerulosclerosis manifested by excessive mesangial matrix deposition in the glomerular tuft with widening of the urinary space and Bowman's capsule thickening. Administration of prophylactic losartan, prophylactic resveratrol and therapeutic resveratrol showed normalization of PAS reaction. Examination of post-DN losartan group on the other hand showed non-significant improvement in glomerulosclerosis index with remains of Bowman's capsule thickening and capillary-to-tuft adhesions. 


\section{Examination of MT stained sections (Fig 5):}

The DN group displayed excessive collagen deposition in the glomeruli, glomerular basement membranes and interstitial tissue. Assessment of the prophylactic and treatment groups showed normal collagen distribution pattern around renal tubules and in the glomeruli except for localized areas of increased collagen deposition in the glomerular capillary tuft of pre-DN losartan group and focal collagen deposition in the Bowman's capsule of post-DN losartan group.

\section{Histomorphometric parameters (Table 2):}

Assessment of glomerular parameters showed significant improvement with prophylactic losartan and resveratrol while the treatment groups on the other hand showed non-significant differences relative to the diabetic group. Regarding tubular parameters, the tubular diameter and tubular epithelial height showed significant increase in preDN losartan, pre-DN resveratrol, and post-DN resveratrol groups, while the bush border length demonstrated significant increase only with preDN losartan.
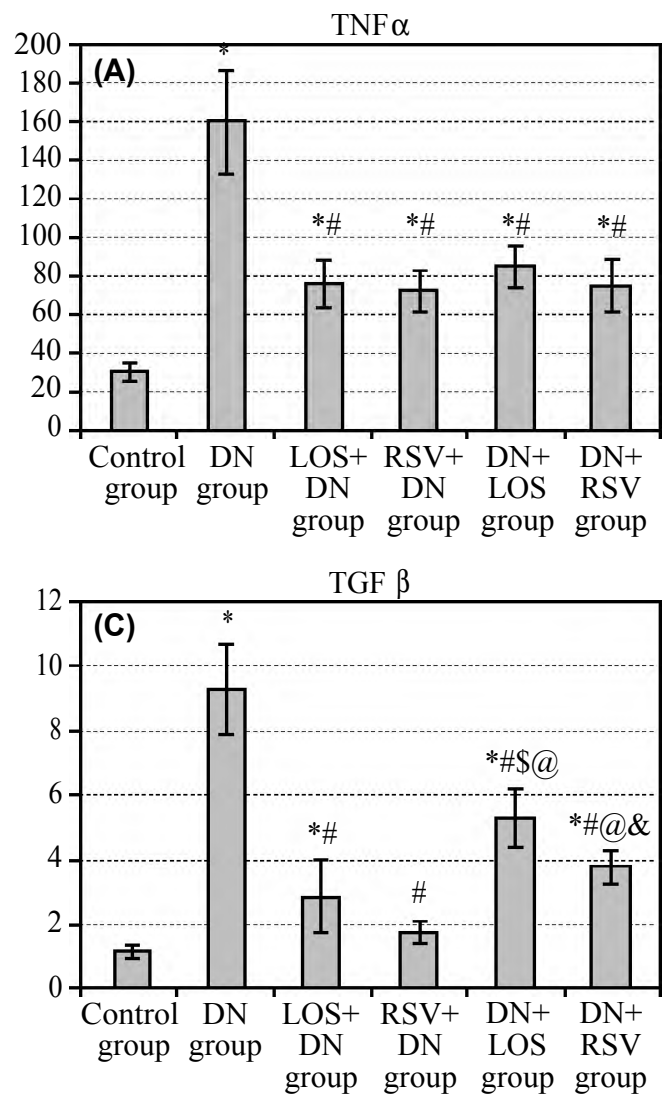

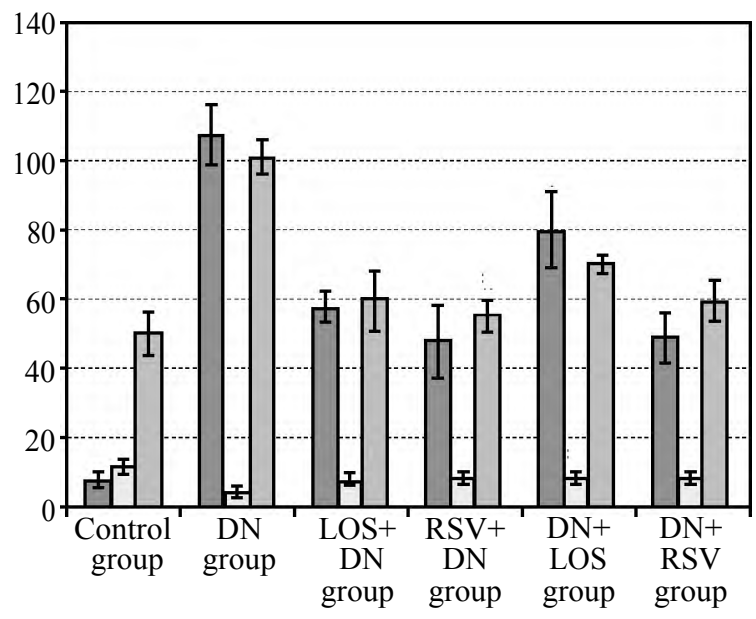

UAEQGFR $\square$ UREA

Fig. (1): Levels of kidney function parameters: Urine albumin excretion (UAE), glomerular filtration rate (GFR), urea in all studied groups. Values are represented as mean \pm S.D., $(n=8)$.

* : Statistically significant compared to control group $(p<0.05)$

\# : Statistically significant compared to diabetic nephropathy group $(p<0.05)$

$\$$ : Statistically significant compared to LOS+DN group $(p<0.05)$.

(a): Statistically significant compared to RSV+DN (NO2-OA) group $(p<0.05)$

\&: Statistically significant compared to DN+LOS group $(p<0.05)$.
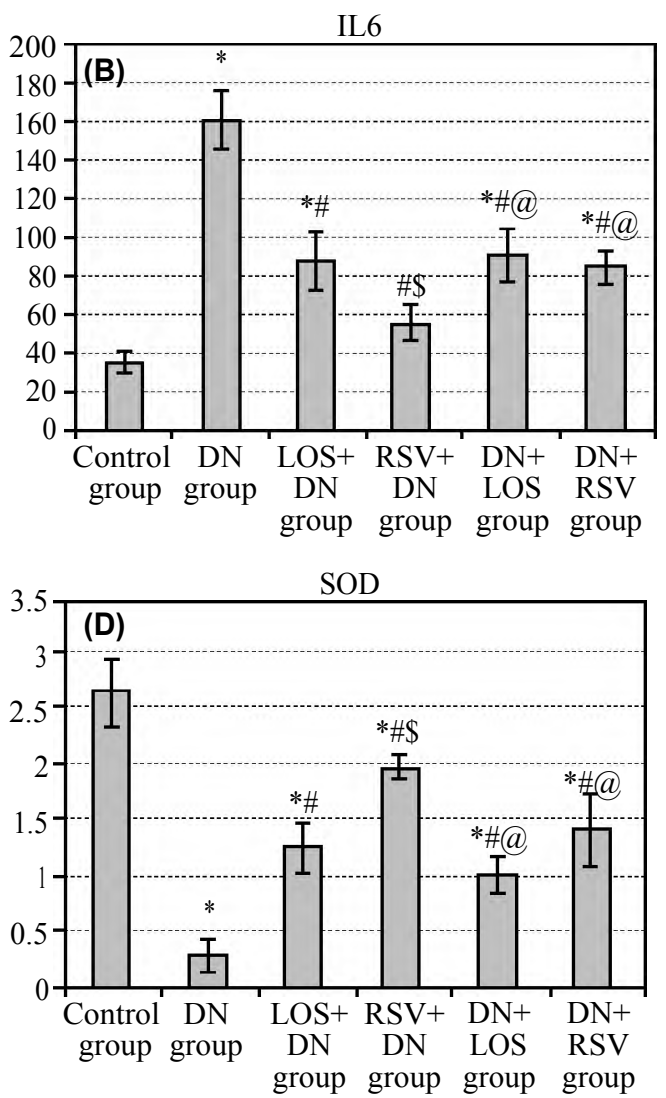

Fig. (2A): Tumor necrosis factor- $\alpha$ (TNF- $\alpha$ ), (B) interleukin 6 (IL6), (C) tissue growth factor- (TGF- $\beta$ ), (D) superoxide dismutase (SOD) among studied groups. Each vertical bar represents the mean \pm SD of 8 animals.

* : Statistically significant compared to corresponding value in control group $(p<0.05)$.

\# : Statistically significant compared to corresponding value in diabetic nephropathy group $(p<0.05)$

$\$$ : Statistically significant compared to corresponding value in LOS+DN group $(p<0.05)$.

@: : Statistically significant compared to corresponding value in $\operatorname{RSV}+\mathrm{DN}$ group $(p<0.05)$.

\&: Statistically significant compared to corresponding value in $\mathrm{DN}+\mathrm{LOS}$ group $(p<0.05)$. 

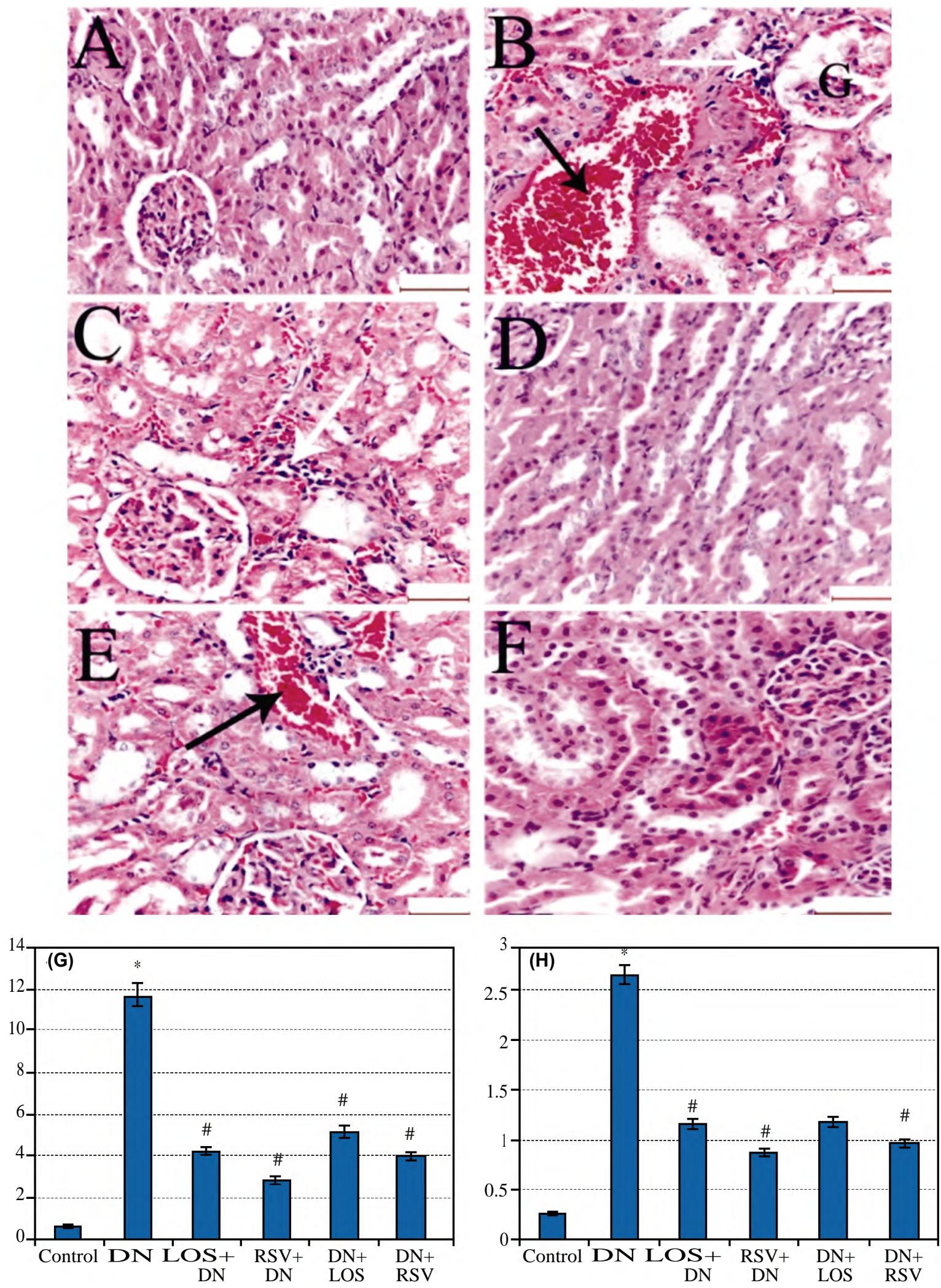

Fig. (3): H\&E stained sections of (A): Control group, (B): DN group showing degenerated segmented glomerulus with widened Bowman's space, large area of congestion and interstitial infiltration, (C): Pre-DN losartan group showing minimal interstitial infiltration, D: Pre-DN resveratrol group showing healthy glomeruli and tubules, E: Post-DN losartan group, (F): Post-DN resveratrol group (Black arrow: Congestion, white arrow: interstitial infiltration) (A, B, C, D, E, F: x 400). (G): Bar chart representing interstitial cell infiltration score in the different studied groups. H: Area percent of congestion in the different studied groups. Each vertical bar represents the mean ${ }_{-;}$SD of 8 animals.

*: Statistically significant compared to corresponding value in control group $(p<0.05)$.

\#: Statistically significant compared to corresponding value in diabetic nephropathy group $(p<0.05)$. 

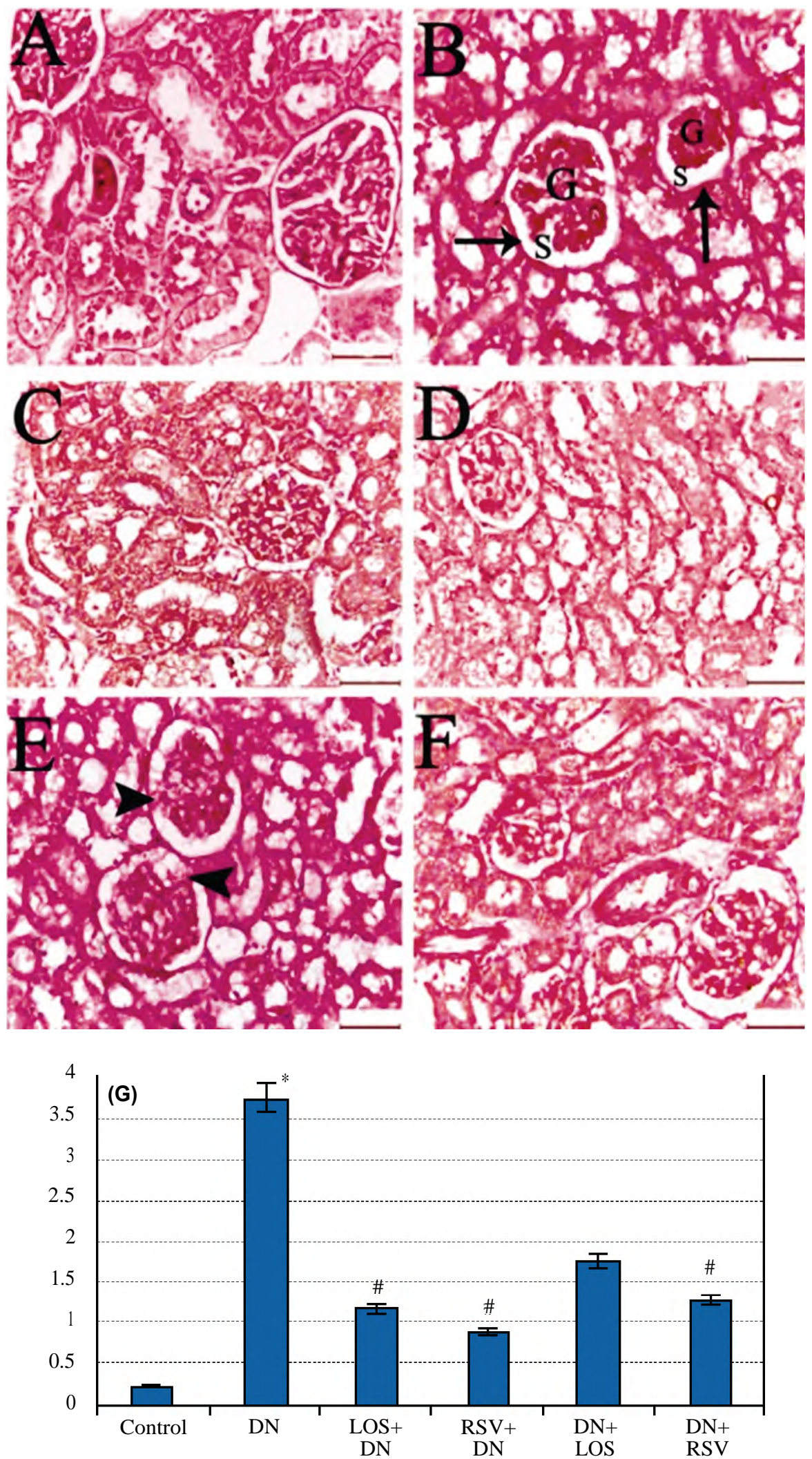

Fig. (4): PAS stained sections of (A): Control group, (B): DN group showing shrunken sclerotic glomeruli (G): With widened urinary space (S) and thickened parietal layer of Bowman's capsule (arrow), (C): Pre-DN losartan group, D: Pre-DN resveratrol group, E: Post-DN losartan group showing remains of tuft-to-capsule adhesions (arrow heads), (F): Post-DN resveratrol group (A, B, C, D, E, F: x400). (G): Bar chart representing glomerulosclerosis index in the different studied groups. Each vertical bar represents the mean $\pm \mathrm{SD}$ of 8 animals.

*: Statistically significant compared to corresponding value in control group $(p<0.05)$.

\#: Statistically significant compared to corresponding value in diabetic nephropathy group $(p<0.05)$. 

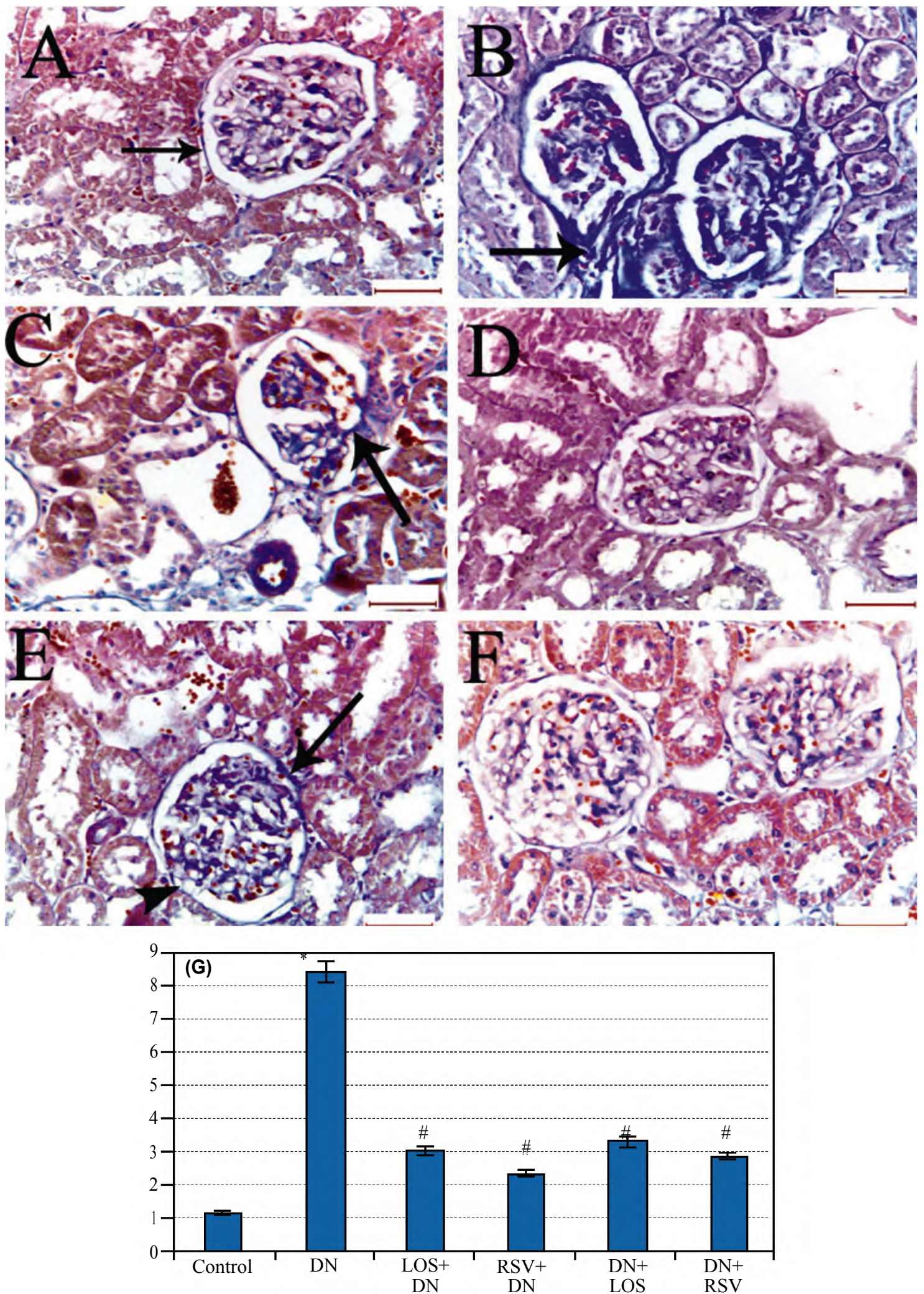

Fig. (5): Masson's trichrome stained sections of (A): Control group, (B): DN group showing features of glomerulosclerosis and peri-glomerular fibrosis (arrow), (C): Pre-DN losartan group showing mild collagen deposition at the root of the glomerular capillary tuft (arrow), (D): Pre-DN resveratrol group, (E): Post-DN losartan group showing mild increase in collagen deposition of the Bowman's capsule parietal layer (arrow) with remains of tuftto-capsule adhesions (arrow head), F: Post-DN resveratrol group (A, B, C, D, E, F: X400). (G): Bar chart representing area percent of collagen in the different studied groups. Each vertical bar represents the mean $\pm \mathrm{SD}$ of 8 animals.

*: Statistically significant compared to control group $(p<0.05)$.

\#: Statistically significant compared to corresponding value in diabetic nephropathy group. 
Table (1): Measurement of fasting serum glucose, systolic blood pressure (SBP) in the studied groups.

\begin{tabular}{lllllll}
\hline & $\begin{array}{c}\text { Control } \\
\text { group }\end{array}$ & $\begin{array}{c}\text { DN } \\
\text { group }\end{array}$ & $\begin{array}{c}\text { LOS+DN } \\
\text { group }\end{array}$ & $\begin{array}{c}\text { RSV+DN } \\
\text { group }\end{array}$ & $\begin{array}{c}\text { DN+LOS } \\
\text { group }\end{array}$ & $\begin{array}{c}\text { DN+RSV } \\
\text { group }\end{array}$ \\
\hline GLUCOSE & $90.80 \pm$ & $226.93 \pm$ & $164.42 \pm$ & $156.67 \pm$ & $186.63 \pm$ & $165.62 \pm$ \\
& 5.17 & $7.37 *$ & $9.82 * \#$ & $11.97 * \#$ & $10.67 * \# \$ @$ & $12.52 * \# \&$ \\
SBP & $107.17 \pm$ & $150.00 \pm$ & $129.00 \pm$ & $128.67 \pm$ & $134.17 \pm$ & $129.00 \pm$ \\
& 4.49 & $7.13 *$ & $6.54 * \#$ & $5.28 * \#$ & $5.34 * \#$ & $6.84 * \#$ \\
\hline
\end{tabular}

* : Statistically significant compared to corresponding value in control group $(p<0.05)$.

\# : Statistically significant compared to corresponding value in diabetic nephropathy group $(p<0.05)$.

$\$$ : Statistically significant compared to corresponding value in LOS+DN group $(p<0.05)$.

@: Statistically significant compared to corresponding value in $\mathrm{RSV}+\mathrm{DN}$ group $(p<0.05)$

\&: Statistically significant compared to corresponding value in DN+LOS group $(p<0.05)$.

Table (2): Histomorphometric parameters.

\begin{tabular}{|c|c|c|c|c|c|c|}
\hline & $\begin{array}{l}\text { Control } \\
\text { group }\end{array}$ & $\begin{array}{l}\mathrm{DN} \\
\text { group }\end{array}$ & $\begin{array}{l}\text { Prophylactic } \\
\text { Losatan }\end{array}$ & $\begin{array}{l}\text { Prophylactic } \\
\text { Resveratrol }\end{array}$ & $\begin{array}{l}\text { Treated } \\
\text { Losatan }\end{array}$ & $\begin{array}{c}\text { Treated } \\
\text { Resveratrol }\end{array}$ \\
\hline $\begin{array}{l}\text { Bowman's } \\
\left.\text { space ( } \mathbf{m}^{2}\right)\end{array}$ & $\begin{array}{l}1645.17 \pm \\
31.30\end{array}$ & $\begin{array}{l}2906.40 \pm \\
12.11 \#\end{array}$ & $\begin{array}{l}2414.01 \pm \\
55.01 *\end{array}$ & $\begin{array}{l}2015.16 \pm \\
11.9^{*}\end{array}$ & $\begin{array}{l}2912.3 \pm \\
71.4 \mathrm{NS}\end{array}$ & $\begin{array}{l}2591.1 \pm \\
27.12 \mathrm{NS}\end{array}$ \\
\hline $\begin{array}{l}\text { Glomerular } \\
\text { area }\left(\mathbf{m}^{2}\right)\end{array}$ & $\begin{array}{l}3011.22 \pm \\
51.71\end{array}$ & $\begin{array}{l}2020.31 \pm \\
21.6 \#\end{array}$ & $\begin{array}{l}2211.21 \pm \\
82.5^{*}\end{array}$ & $\begin{array}{l}2532.1 \pm \\
72.32 * *\end{array}$ & $\begin{array}{l}2155.4 \pm \\
31.1 \mathrm{NS}\end{array}$ & $\begin{array}{l}2041.2 \pm \\
50.01 \mathrm{NS}\end{array}$ \\
\hline $\begin{array}{l}\text { Tubular Epithelial } \\
\text { height }(\mathrm{m})\end{array}$ & $\begin{array}{l}18.12 \pm \\
1.4\end{array}$ & $\begin{array}{l}7.93 \pm \\
2.16 \#\end{array}$ & $\begin{array}{l}11.07 \pm \\
2.6^{*}\end{array}$ & $\begin{array}{l}14.51 \pm \\
3.5^{*}\end{array}$ & $\begin{array}{l}8.17 \pm \\
2.43 \mathrm{NS}\end{array}$ & $\begin{array}{l}10.12 \pm \\
4.71^{*}\end{array}$ \\
\hline $\begin{array}{l}\text { Length of the brush } \\
\text { border }(\mathrm{m})\end{array}$ & $\begin{array}{l}0.71 \pm \\
0.08\end{array}$ & $\begin{array}{l}0.32 \pm \\
0.03 \#\end{array}$ & $\begin{array}{l}0.54 \pm \\
0.02^{*}\end{array}$ & $\begin{array}{l}0.52 \pm \\
0.04 \mathrm{NS}\end{array}$ & $\begin{array}{l}0.53 \pm \\
0.09 \mathrm{NS}\end{array}$ & $\begin{array}{l}0.37 \pm \\
0.05 \mathrm{NS}\end{array}$ \\
\hline
\end{tabular}

\section{Discussion}

In this study, we demonstrated the protective and therapeutic effect of resveratrol in improving the renal damage associated with diabetic nephropathy in comparison with losartan as a therapeutic regimen in diabetic nephropathy.

The development of diabetic nephropathy in this study was observed by significant deterioration in the kidney function manifested by increased urea and UAE and decreased GFR associated with glomerulosclerosis and interstitial fibrosis.

This observed renal injury was associated with hyperglycemia and elevated systolic blood pressure, these findings supports the results reported that hyperglycemia and hypertension are the major initiators of metabolic and hemodynamic changes in diabetic renal disease, and that progression of DN correlates closely with level of hyperglycemia [3].

Our results showed significant increase in serum concentrations of inflammatory cytokines, IL-6 and TNF- $\alpha$ and decrease in antioxidant SOD in diabetic nephropathy models. This supports the evidence that diabetic nephropathy is a complex disease linking hemodynamic and metabolic path- ways with oxidative stress, systemic inflammation and cytokines [18]

Recent studies have revealed up-regulation of many inflammatory molecules in diabetic patients with progressive rise in their concentrations as DN progresses. Thus, the anti-inflammatory strategy can be a promising therapeutic approach in diabetic patients $[1 \mathbf{1 9 , 2 0}]$. Furthermore, increasing evidence indicates that disruption of mitochondrial bioenergetics and oxidative stress may have a major role in the development and progression of DN [21]

The diabetic effect of elevated inflammatory cytokines and oxidative stress markers in the current study is further confirmed by increased inflammatory cellular infiltration in the DN groups.

Complementary to renal injury signs, renal fibrosis presented by increased glomerular and interstitial collagen deposition was also observed in DN rats. These pathological findings are supported by significant elevation of profibrotic TGF$\beta$ levels.

TGF- $\beta$ gene and protein levels have been reported to be significantly elevated in tubuloglomerular interstitium of diabetic patients and animals [22] 
The current study demonstrated the effects of resveratrol in comparison with losartan in a trial to encourage its use as an evident antidiabetic drug.

Prophylactic resveratrol showed a renoprotective effect by improving the kidney function parameters as urea, UAE and GFR as well as the histopathological features of diabetic nephropathy.

Furthermore, resveratrol-treated rats showed the same beneficial effect on kidney function compared to losartan. It is worth noting that proteinuria apart from other kidney function was significantly improved in resveratrol groups relative to losartan groups.

Resveratrol has previously showed protective effects on renal damage associated with experimentally induced type 1 diabetes by improving functional and histopathological abnormalities [23] In another study, resveratrol seemed to have a renoprotective effect on diabetic kidney, and being an herbal drug, it was advised to be used for earlystage diabetes before developing nephropathy as a prophylactic therapy [24]. Those studies illustrated the protective effects of resveratrol on diabetic kidney before induction of diabetic nephropathy, they didn't investigate its beneficial effects after developing diabetic nephropathy, which is one of the main concerns in this study.

The observed effects on kidney function in this current study can be partially attributed to the significant improvement in glucose and systolic blood pressure levels. Despite the non-significant hypoglycemic effect between prophylactic or curable resveratrol, yet, resveratrol still has a significant hypoglycemic effect compared to losartan.

Several previous studies have reported the antihyperglycemic effect of resveratrol and its modulatory effect on insulin resistance shown by reduced blood glucose levels and delay in the time for peak postprandial glucose in patients receiving resveratrol for 4 weeks [25,26]. Effects of prophylactic and curable resveratrol on the mechanisms underlying diabetic nephropathy were also studied.

Our results are also concordant with the previous reports [20] highlighting the implication of chronic inflammation in the pathogenesis of diabetic nephropathy.

Resveratrol showed a beneficial effect by lowering the levels of the inflammatory cytokines TNF $\alpha$ and IL6 before and after development of DN. In comparison with losartan, prophylactic resveratrol showed more significant reduction of IL6 levels.
Previous studies reported that resveratrol reduced inflammation and counteracted the increased levels of proinflammatory biochemical markers, such as TNF $\alpha$, IL-1 $\beta$ and IL6 in several animal models including obesity- [27] and chemicallyinduced diabetes [28] and induced hypertension [29].

RSV has been shown to induce anti-inflammatory effect in DM mainly via the inhibition of the nuclear factor NF- $\kappa \mathrm{B}$ pathway. NF- $\kappa \mathrm{B}$ is a proinflammatory master switch, which activates proinflammatory cytokines gene expression. Production and release of proinflammatory cytokines and chemokines induce ROS production and further increase tissue oxidative stress resulting in a vicious cycle of inflammation and oxidative stress [30]

Rise of the antioxidative enzyme SOD was observed after administration of resveratrol in this study. Interestingly, this effect was more marked in the prophylactic group than the therapeutic group. This result emphasizes the role of resveratrol in oxidative stress modulation in diabetic nephropathy models. Resveratrol has been observed to be more effective than losartan in ameliorating oxidative stress associated with DM especially in prophylactic model.

Resveratrol can improve functional and histopathological abnormalities in diabetic kidney, possibly by attenuating the oxidative stress and normalization of the Mn-SOD dysfunction and by partial improvement of the glucose and lipid metabolism [31]

The effect of resveratrol on fibrosis associated with DN was also investigated. To our knowledge, there is a lack of studies concerned with this issue. This work showed that both prophylactic and therapeutic resveratrol significantly improved glomerulosclerosis and interstitial fibrosis and also significantly decreased the profibrotic TGF- $\beta$ level. All these findings support the antifibrotic effect of resveratrol that consequently improve renal damage. Administration of resveratrol before DN has a significant improving effect in TGF- $\beta$ in relation to its administration after DN. Interestingly; resveratrol was found to be more effective in ameliorating fibrosis than losartan.

\section{Conclusion:}

Data from the present study suggested that resveratrol can offer partial protection and regression of renal injury associated with diabetic nephropathy. These results may be attributed to its antiinflammatory, antioxidant as well as antifibrotic 
effects, in addition to its beneficial effects on metabolic and hemodynamic states. Resveratrol was found to be more beneficial than losartan, especially when used as a prophylactic regimen. This highlights the beneficial role of resveratrol as a complementary regimen in the prophylaxis and treatment of diabetic nephropathy.

\section{Declaration of interest:}

The authors declare no conflict of interest.

There is no source of funding.

\section{References}

1- HU F.B.: Prevention of diabetes and cardiovascular disease among prediabetic individuals: Lifestyle versus drug interventions. The European Journal of Cardiovascular Prevention \& Rehabilitation, 18 (6): 810-2, 2011.

2- MIMA A.: Inflammation and oxidative stress in diabetic nephropathy: New insights on its inhibition as new therapeutic targets. Journal of Diabetes Research, 248563, 2013.

3- FORBES J.M. and COOPER M.E.: Mechanisms of diabetic complications. Physiological Reviews, 93: 137$188,2013$.

4- RASK-MADSEN C. and KING G.L.: Vascular complications of diabetes: Mechanisms of injury and protective factors. Cell Metabolism, 17: 20-33, 2013.

5- MAUER M., ZINMAN B., GARDINER R., et al.: Renal and retinal effects of enalapril and losartan in type 1 diabetes. New England Journal of Medicine, 361 (1): 4051, 2009.

6- KOGA C., ANDRADE J., FERRUZZI M., et al.: Stability of Trans-Resveratrol Encapsulated in a Protein Matrix Produced Using Spray Drying to UV Light Stress and Simulated Gastro-Intestinal Digestion. Journal of Food Science, 81 (2): C292-300, 2016.

7- GONZALEZ-RODRIGUEZ A., SANTAMARIA B., MAS-GUTIERREZ J.A., et al.: Resveratrol treatment restores peripheral insulin sensitivity in diabetic mice in a sirtl-independent manner. Molecular Nutrition \& Food Research, 59: 1431-42, 2015.

8- BHATT J.K., THOMAS S. and NANJAN M.J.: Resveratrol supplementation improves glycemic control in type 2 diabetes mellitus. Nutrition Research, 32 (7): $537-41,2012$.

9- ZHU X., WU C., QIU S., et al.: Effects of resveratrol on glucose control and insulin sensitivity in subjects with type 2 diabetes: Systematic review and metaanalysis. Nutrition \& Metabolism, 14: 60, 2017.

10- THAZHATH S.S., WU T., BOUND M.J., et al.: Administration of resveratrol for 5 wk has no effect on glucagon-like peptide 1 secretion, gastric emptying, or glycemic control in type 2 diabetes: A randomized controlled trial. The American Journal of Clinical Nutrition, 103: 66-70, 2016.

11-LIU Y., JIA Z., LIU S., et al.: Combined losartan and nitro-oleic acid remarkably improves diabetic nephropathy in mice. American journal of physiology. Renal physiology, 305 (11): F1555-62, 2013.

12-SHARMA S., ANJANEYULU M., KULKARNI S., et al.: Resveratrol, a polyphenolic phytoalexin, attenuates diabetic nephropathy in rats. Pharmacology, 76 (2): 6975, 2006.

13-GOJO A., UTSUNOMIYA K., TANIGUCHI K., et al.: The Rho-kinase inhibitor, fasudil, attenuates diabetic nephropathy in streptozotocin-induced diabetic rats. European Journal of Pharmacology, 568 (1-3): 242-7, 2007.

14- SINGH R., SINGH A.K., and LEEHEY D.J.: A novel mechanism for angiotensin II formation in streptozotocindiabetic rat glomeruli. American Journal of PhysiologyRenal Physiology, 288 (6): F1183-F1190, 2005.

15-XIONG Z.K., LANG J., XU G., et al.: Excessive levels of nitric oxide in rat model of Parkinson's disease induced by rotenone. Experimental and therapeutic medicine, 9 (2): 553-558, 2015.

16-MARIC C., SANDBERG K. and HINOJOSA-LABORDE C.: Glomerulosclerosis and tubulointerstitial fibrosis are attenuated with $17 \mathrm{j} 3$-estradiol in the aging Dahl salt sensitive rat. Journal of the American Society of Nephrology, 15 (6): 1546-1556, 2004.

17-SAITO T., SUMITHRAN E., GLASGOW E.F., et al.: The enhancement of aminonucleoside nephrosis by the coadministration of protamine. Kidney international, 32 (5): 691-699,1987.

18-SATIRAPOJ B. and ADLER S.: Comprehensive approach to diabetic nephropathy. Kidney Research and Clinical Practice, 33 (3): 121-31, (2014).

19-DONATE-CORREA J., MARTIN-Ni SIEZ E., MUROSDE-FUENTES M., et al.: Inflammatory Cytokines in Diabetic Nephropathy. Journal of Diabetes Research, Article ID 948417, 9 pages, 2015.

20- PESTANA R.M., DOMINGUETI C.P., DUARTE R.C., et al.: Cytokines profile and its correlation with endothelial damage and oxidative stress in patients with type 1 diabetes mellitus and nephropathy. Immunologic Research, 64 (4): 951-60,2016.

21- SIVITZ W. and YOREK M.: Mitochondrial dysfunction in diabetes: From molecular mechanisms to functional significance and therapeutic opportunities. Antioxidants \& Redox Signaling, 12 (4): 537-77, 2010.

22-YAMAGISHI S. and MATSUI T.: Advanced glycation end products, oxidative stress and diabetic nephropathy. Oxidative Medicine and Cellular Longevity, 3 (2): 1018, 2010 .

23-EZEL T., KOCYIGIT Y., DEVECI E., et al.: Biochemical and histopathological investigation of reveratrol. Gliclazide, and losartan protective effects on renal damage in a diabetic rat model. Analytical and Quantitative Cytopathology and Histopathology, 37 (3): 187-98, 2015.

24- ABDALLAH M. and EL-DEBAKEY F.: Prophylactic Resveratrol and Telmisartan Combination Ameliorates Experimentally-Induced Diabetic Nephropathy in Rats, Focus on the Pro-Sclerotic Cytokine, Transforming Growth Factor-PH (TGF -(31). Medical Journal of Cairo University, 78(2): 705-713, 2010. 
25- PALSAMY P., SIVAKUMAR S., SUBRAMANIAN S., et al.: Resveratrol attenuates hyperglycemia-mediated oxidative stress, proinflammatory cytokines and protects hepatocytes ultrastructure in streptozotocin-nicotinamideinduced experimental diabetic rats, Chemico-Biological Interactions, 186 (2): 200-210, 2010.

26- BRASNYÓ P.1., MOLNÁR G.A., MOHÁS M. et al.: Resveratrol improves insulin sensitivity, reduces oxidative stress and activates the Akt pathway in type 2 diabetic patients. British Journal of Nutrition, 106 (3): 383-9, 2011.

27- RIVERA L., MORÓN R., ZARZUELO A., et al.: Longterm resveratrol administration reduces metabolic disturbances and lowers blood pressure in obese Zucker rats. Biochemical Pharmacology, 77 (6): 1053-63, 2009.

28- KUMAR A. and SHARMA S.: NF-kappaB inhibitory action of resveratrol: A probable mechanism of neuroprotection in experimental diabetic neuropathy. Biochemical and Biophysical Research Communications, 394 (2): 360-5, 2010.

29- INANAGA K., ICHIKI T., MATSUURA H., et al.: Resveratrol attenuates angiotensin II-induced interleukin6 expression and perivascular fibrosis. Hypertension Research, 32 (6): 466-71, 2009.

30- LAWRENCE T.: The nuclear factor NF- $\kappa$ B pathway in inflammation. Cold Spring Harbor Perspectives in Biology, 1 (6), 2009.

31- KITADA M., KUME S., IMAIZUMI N., et al.: Resveratrol improves oxidative stress and protects against diabetic nephropathy through normalization of Mn-SOD dysfunction in AMPK/SIRT 1-independent pathway. Diabetes, 60 (2): 634-43, 2011.

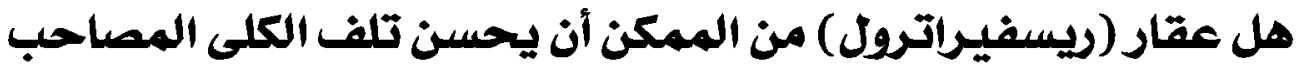

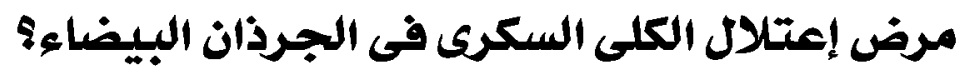

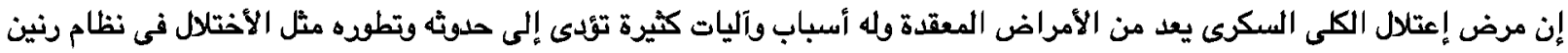

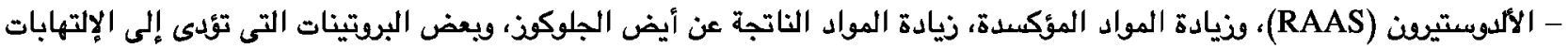

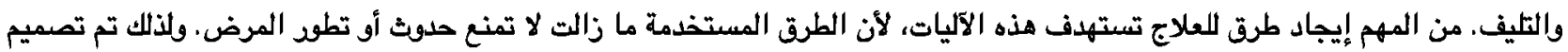

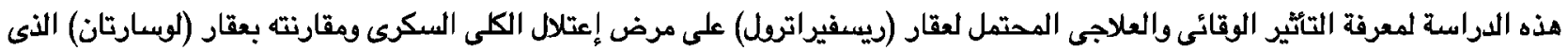

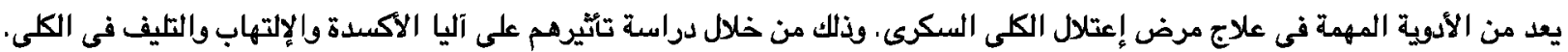
تمت الدراسة بإستخدام ^ع من ذكور الفئران المعملية البيضاء، وتم تقسيمها إلى 7 مجموعات: المجموعة الضابطة، مجموعة إعتلال الكلى

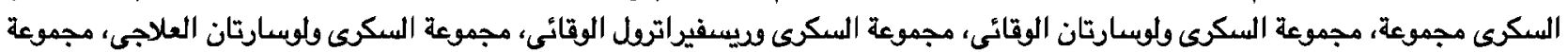

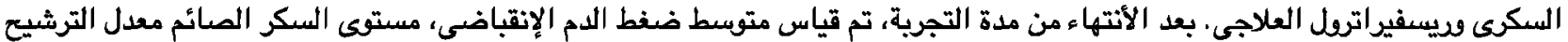
الكبيبى GFR البولينة، وإفراز الزلال البولى، هستوى الكلى لتحليل الهيستوباثولوجى.

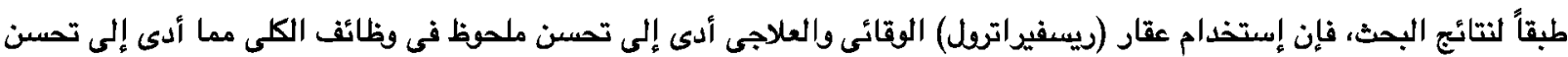

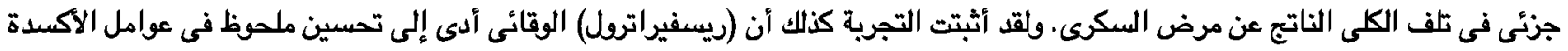
والتلف والإلتهاب مقارنة بإستخدامه كعلاج، مع أفضليته فى بعض النغ النتائج على (لوسارتان).

ويستتج من هذا البحث أن إستخدام عقار (ريسفيراترول) تد يساعد فى الوقاية والعلاج من مرض إعتلال الكلى السكرى وينصح به كعقار مساعد ضمن نظام العلاج المتبع للمريض. 